\title{
CENTRES OF SYMMETRIC CELLULAR ALGEBRAS
}

\author{
YANBO LI
}

(Received 15 December 2009)

\begin{abstract}
Let $R$ be an integral domain and $A$ a symmetric cellular algebra over $R$ with a cellular basis $\left\{C_{S, T}^{\lambda} \mid \lambda \in\right.$ $\Lambda, S, T \in M(\lambda)\}$. We construct an ideal $L(A)$ of the centre of $A$ and prove that $L(A)$ contains the socalled Higman ideal. When $R$ is a field, we prove that the dimension of $L(A)$ is not less than the number of nonisomorphic simple $A$-modules.
\end{abstract}

2000 Mathematics subject classification: primary 16U70; secondary 16G30, 20 C08.

Keywords and phrases: symmetric cellular algebras, centre.

\section{Introduction}

In 1996, Graham and Lehrer [11] introduced cellular algebras in order to provide a systematic framework for studying the representation theory of a class of algebras. By the theory of cellular algebras, one can parameterize simple modules for a finitedimensional cellular algebra by methods in linear algebra. Many classes of algebras from mathematics and physics are found to be cellular, including Hecke algebras of finite type, Ariki-Koike algebras, $q$-Schur algebras, Brauer algebras, Temperley-Lieb algebras, cyclotomic Temperley-Lieb algebras, partition algebras, Birman-Wenzl algebras and so on; see $[8,11,16-18]$ for details.

There are many papers on centres of Hecke algebras of finite type, which are all cellular algebras [8]. In [13], Jones found bases for centres of Hecke algebras of type A over $\mathbb{Q}\left[q, q^{-1}\right]$, where $q$ is an indeterminant. This basis is an analogue of conjugacy class sums in a group algebra. In [10], Geck and Rouquier found bases for the centres of generic Hecke algebras over $\mathbb{Z}\left[q, q^{-1}\right]$ with $q$ an indeterminant. However, it is not easy to write the basis explicitly. Then one should ask, is there any basis which can be written explicitly? In [4], Francis gave an integral minimal basis for the centre of a Hecke algebra. Then in [5], he used the minimal basis approach to provide an entirely combinatorial way of describing and calculating elements of the minimal basis for the centre of an Iwahori-Hecke algebra. In [7], Francis and Jones found an explicit nonrecursive expression for the coefficients appearing in these linear combinations

This work was partially supported by the Research Fund of Doctor Program of Higher Education, Ministry of Education of China.

(C) 2010 Australian Mathematical Publishing Association Inc. 0004-9727/2010 \$16.00 
for the Hecke algebras of type A. The relations between the so-called Jucys-Murphy elements and centres of Hecke algebras are also investigated widely. In [3], Dipper and James conjectured that the centre of a Hecke algebra of type A consists of symmetric polynomials in the Jucys-Murphy elements. This conjecture was proved by Francis and Graham [6] in 2006. An analogous conjecture for Ariki-Koike algebras is still open.

Jeong et al. [12] firstly studied centres of semisimple Hecke algebras of some finite types using the cellular basis. The fact that Hecke algebras of finite type are all cellular led us to consider how to describe the centres of cellular algebras in general. Clearly, most of the approaches for studying Hecke algebras cannot be used directly for cellular algebras, since we have no Weyl group structure to use. Thus we have to look for some new methods. In fact, the symmetry of Hecke algebras provides us with a way to do so. In the present paper, we consider the centres of symmetric cellular algebras. Note that Hecke algebras of all finite types [8], Ariki-Koike algebras with invertible parameters [15] and Khovanov's diagram algebras [1] are all symmetric.

In order to describe our result exactly, we fix some notation first. Let $A$ be a symmetric cellular $R$-algebra with a nondegenerate symmetric bilinear form $f$ : $A \times A \rightarrow R$. Then $f$ determines a map $\tau: A \rightarrow R$ which is defined by $\tau(a)=f(a, 1)$ for every $a \in A$. We call the map $\tau$ a symmetrizing trace. Denote by

$$
\left\{D_{S, T}^{\lambda} \mid S, T \in M(\lambda), \lambda \in \Lambda\right\}
$$

the dual basis determined by $\tau$. Let

$$
H(A)=\left\{\sum_{\lambda \in \Lambda, S, T \in M(\lambda)} C_{S, T}^{\lambda} a D_{S, T}^{\lambda} \mid a \in A\right\} ;
$$

this is the Higman ideal of $Z(A)$. For any $\lambda \in \Lambda$ and $T \in M(\lambda)$, set $e_{\lambda}=$ $\sum_{S \in M(\lambda)} C_{S, T}^{\lambda} D_{S, T}^{\lambda}$, where $e_{\lambda}$ is independent of $T$ and $L(A)=\left\{\sum_{\lambda \in \Lambda} r_{\lambda} e_{\lambda} \mid r_{\lambda} \in R\right\}$. Then we have the following theorem.

THEOREM 1.1. Let $A$ be a symmetric cellular algebra with a cellular basis $\left\{C_{S, T}^{\lambda} \mid\right.$ $S, T \in M(\lambda), \lambda \in \Lambda\}$ and a dual basis $\left\{D_{S, T}^{\lambda} \mid S, T \in M(\lambda), \lambda \in \Lambda\right\}$ determined by a symmetrizing trace $\tau$. Then $L(A)$ is an ideal of $Z(A)$ containing the Higman ideal.

It is helpful to note that, when $R$ is a field, the dimension of $L(A)$ is not less than the number of nonisomorphic simple $A$-modules (see Proposition 3.3 below). Therefore, $L(A)$ is the whole centre provided that $A$ is semisimple. In particular, we give a complete set of primitive orthogonal idempotents of a symmetric cellular algebra when the algebra is semisimple. Note that Dipper and James did the same thing for a Hecke algebra of type $\mathrm{A}$ in [3].

\section{Preliminaries}

In this section, we first recall some basic results on symmetric algebras and cellular algebras, which are needed in the following sections. The so-called Higman ideal is also described. References for this section are the books [2, 9]. 
Let $R$ be a commutative ring with identity and $A$ an associative $R$-algebra. Assume that $A$ is finitely generated and free as an $R$-module. Suppose that there exists an $R$-bilinear map $f: A \times A \rightarrow R$. We say that $f$ is nondegenerate if the determinant of the matrix $\left(f\left(a_{i}, a_{j}\right)\right)_{a_{i}, a_{j} \in B}$ is a unit in $R$ for some $R$-basis $B$ of $A$. We say $f$ is associative if $f(a b, c)=f(a, b c)$ for all $a, b, c \in A$, and symmetric if $f(a, b)=$ $f(b, a)$ for all $a, b \in A$.

Definition 2.1. An $R$-algebra $A$ is said to be symmetric if there is a nondegenerate associative symmetric bilinear form $f$ on $A$. Define an $R$-linear map $\tau: A \rightarrow R$ by $\tau(a)=f(a, 1)$. We call $\tau$ a symmetrizing trace.

Let $A$ be a symmetric algebra with a basis $B=\left\{a_{i} \mid i=1, \ldots, n\right\}$ and $\tau$ a symmetrizing trace. Denote by $D=\left\{D_{i} \mid i=1, \ldots, n\right\}$ the basis determined by the requirement that $\tau\left(D_{j} a_{i}\right)=\delta_{i j}$ for all $i, j=1, \ldots, n$. We will call $D$ the dual basis of $B$. For arbitrary $1 \leq i, j \leq n$, we write $a_{i} a_{j}=\sum_{k} r_{i j k} a_{k}$, where $r_{i j k} \in R$. Fixing a $\tau$ for $A$, we proved the following lemma in [14].

LEMMA 2.2. If $A$ is a symmetric algebra with a basis $B$ and the dual basis $D$, then $a_{i} D_{j}=\sum_{k} r_{k i j} D_{k}$ and $D_{i} a_{j}=\sum_{k} r_{j k i} D_{k}$.

It is well known that $\left\{\sum_{i} D_{i} a a_{i} \mid a \in A\right\}$ is an ideal of the centre of $A$, which is called the Higman ideal [2]. We provide an elementary proof of this fact.

Proposition 2.3. Let A be a symmetric algebra with a basis $B$ and the dual basis $D$. Then $\left\{\sum_{i} D_{i} a a_{i} \mid a \in A\right\}$ is an ideal of the centre of $A$.

PROOF. For arbitrary $a_{j} \in B$ and $a \in A$, by Lemma 2.2,

$$
\sum_{i} D_{i} a a_{i} a_{j}=\sum_{i, k} r_{i j k} D_{i} a a_{k}
$$

and

$$
\sum_{i} a_{j} D_{i} a a_{i}=\sum_{i, k} r_{k j i} D_{k} a a_{i}
$$

Obviously, the right-hand sides of the above two equations are equal. Then $\left\{\sum_{i} D_{i} a a_{i} \mid a \in A\right\} \subseteq Z(A)$. It is clear that the set is an ideal of the centre of $A$.

Lemma 2.4 [14, Proposition 2.3]. Suppose that $A$ is a symmetric R-algebra with a basis $\left\{a_{i} \mid i=1, \ldots, n\right\}$. Let $\tau, \tau^{\prime}$ be two symmetrizing traces. Denote by $\left\{D_{i} \mid i=\right.$ $1, \ldots, n\}$ the dual basis of $B$ determined by $\tau$ and by $\left\{D_{i}^{\prime} \mid i=1, \ldots, n\right\}$ the dual basis determined by $\tau^{\prime}$. Then, for $1 \leq i \leq n$,

$$
D_{i}^{\prime}=\sum_{j=1}^{n} \tau\left(a_{j} D_{i}^{\prime}\right) D_{j} .
$$

We now recall the definition of cellular algebras introduced by Graham and Lehrer [11] and some well-known results. 
Definition 2.5 [11, Definition 1.1]. Let $R$ be a commutative ring with identity. An associative unital $R$-algebra is called a cellular algebra with cell datum $(\Lambda, M, C, i)$ if the following conditions are satisfied.

(C1) The finite set $\Lambda$ is a poset. Associated with each $\lambda \in \Lambda$, there is a finite set $M(\lambda)$. The algebra $A$ has an $R$-basis $\left\{C_{S, T}^{\lambda} \mid S, T \in M(\lambda), \lambda \in \Lambda\right\}$.

(C2) The map $i$ is an $R$-linear anti-automorphism of $A$ with $i^{2}=\mathrm{id}$ which sends $C_{S, T}^{\lambda}$ to $C_{T, S}^{\lambda}$.

(C3) If $\lambda \in \Lambda$ and $S, T \in M(\lambda)$, then, for any element $a \in A$,

$$
a C_{S, T}^{\lambda} \equiv \sum_{S^{\prime} \in M(\lambda)} r_{a}\left(S^{\prime}, S\right) C_{S^{\prime}, T}^{\lambda} \quad \bmod A(<\lambda),
$$

where $r_{a}\left(S^{\prime}, S\right) \in R$ is independent of $T$ and where $A(<\lambda)$ is the $R$-submodule of $A$ generated by $\left\{C_{S^{\prime \prime}, T^{\prime \prime}}^{\mu} \mid S^{\prime \prime}, T^{\prime \prime} \in M(\mu), \mu<\lambda\right\}$.

On applying $i$ to the equation in (C3) we obtain

$\left(\mathrm{C}^{\prime}\right) C_{T, S^{\prime}}^{\lambda} i(a) \equiv \sum_{S^{\prime} \in M(\lambda)} r_{a}\left(S^{\prime}, S\right) C_{T, S^{\prime}}^{\lambda} \bmod A(<\lambda)$.

By Definition 2.5, it is easy to check that

$$
C_{S, S}^{\lambda} C_{T, T}^{\lambda} \equiv \Phi(S, T) C_{S, T}^{\lambda} \bmod A(<\lambda),
$$

where $\Phi(S, T) \in R$ depends only on $S$ and $T$.

Let $A$ be a cellular algebra with cell datum $(\Lambda, M, C, i)$. We recall the definition of cell modules.

Definition 2.6 [11, Definition 2.1]. For each $\lambda \in \Lambda$, define the left $A$-module $W(\lambda)$ as follows: $W(\lambda)$ is a free $R$-module with basis $\left\{C_{S} \mid S \in M(\lambda)\right\}$ and $A$-action defined by

$$
a C_{S}=\sum_{S^{\prime} \in M(\lambda)} r_{a}\left(S^{\prime}, S\right) C_{S^{\prime}} \quad \text { for } a \in A, S \in M(\lambda),
$$

where $r_{a}\left(S^{\prime}, S\right)$ is the element of $R$ defined in (C3).

For a cell module $W(\lambda)$, define a bilinear form $\Phi_{\lambda}: W(\lambda) \times W(\lambda) \rightarrow R$ by $\Phi_{\lambda}\left(C_{S}, C_{T}\right)=\Phi(S, T)$ and define

$$
\operatorname{rad}(\lambda):=\left\{x \in W(\lambda) \mid \Phi_{\lambda}(x, y)=0 \text { for all } y \in W(\lambda)\right\}
$$

Graham and Lehrer proved the following results in [11].

THEOREM 2.7. Let $K$ be a field and $A$ a finite-dimensional cellular algebra. For $\lambda \in \Lambda$, denote the A-module $W(\lambda) / \operatorname{rad}(\lambda)$ by $L_{\lambda}$. Let $\Lambda_{0}=\left\{\lambda \in \Lambda \mid \Phi_{\lambda} \neq 0\right\}$. Then $\left\{L_{\lambda} \mid \lambda \in \Lambda_{0}\right\}$ is a complete set of (representatives of equivalence classes of) absolutely simple A-modules. 


\section{Centres of symmetric cellular algebras}

Let $A$ be a symmetric cellular algebra with a cell datum $(\Lambda, M, C, i)$. Denote the dual basis by $D=\left\{D_{S, T}^{\lambda} \mid S, T \in M(\lambda), \lambda \in \Lambda\right\}$, which satisfies

$$
\tau\left(C_{S, T}^{\lambda} D_{U, V}^{\mu}\right)= \begin{cases}1 & \text { for } \lambda=\mu, S=U, T=V \\ 0 & \text { otherwise. }\end{cases}
$$

For any $\lambda, \mu \in \Lambda, S, T \in M(\lambda), U, V \in M(\mu)$, write

$$
C_{S, T}^{\lambda} C_{U, V}^{\mu}=\sum_{\epsilon \in \Lambda, X, Y \in M(\epsilon)} r_{(S, T, \lambda),(U, V, \mu),(X, Y, \epsilon)} C_{X, Y}^{\epsilon} .
$$

In [14] we proved the following lemma.

LEMMA 3.1. Let $A$ be a symmetric cellular algebra with a basis $B$. Let $D$ be the dual basis determined by a given $\tau$. For arbitrary $\lambda, \mu \in \Lambda$ and $S, T, P, Q \in M(\lambda)$, $U, V \in M(\mu)$, the following equations hold.

(1) $D_{U, V}^{\mu} C_{S, T}^{\lambda}=\sum_{\epsilon \in \Lambda, X, Y \in M(\epsilon)} r_{(S, T, \lambda),(X, Y, \epsilon),(U, V, \mu)} D_{X, Y}^{\epsilon}$.

(2) $C_{S, T}^{\lambda} D_{U, V}^{\mu}=\sum_{\epsilon \in \Lambda, X, Y \in M(\epsilon)} r_{(X, Y, \epsilon),(S, T, \lambda),(U, V, \mu)} D_{X, Y}^{\epsilon} \cdot$

(3) $C_{S, T}^{\lambda} D_{S, T}^{\lambda}=C_{S, P}^{\lambda} D_{S, P}^{\lambda}$.

(4) $D_{S, T}^{\lambda} C_{S, T}^{\lambda}=D_{P, T}^{\lambda} C_{P, T}^{\lambda}$.

(5) $C_{S, T}^{\lambda} D_{P, Q}^{\lambda}=0$ if $T \neq Q$.

(6) $D_{P, Q}^{\lambda} C_{S, T}^{\lambda}=0$ if $P \neq S$.

(7) $C_{S, T}^{\lambda} D_{U, V}^{\mu}=0$ if $\mu \not \lambda$.

(8) $D_{U, V}^{\mu} C_{S, T}^{\lambda}=0$ if $\mu \not \Sigma \lambda$.

Let $A$ be a symmetric cellular algebra with a symmetrizing trace $\tau$. The dual basis $\left\{D_{S, T}^{\lambda} \mid S, T \in M(\lambda), \lambda \in \Lambda\right\}$ is determined by $\tau$. Then the Higman ideal is

$$
H(A)=\left\{\sum_{\lambda \in \Lambda, S, T \in M(\lambda)} C_{S, T}^{\lambda} a D_{S, T}^{\lambda} \mid a \in A\right\} .
$$

For any $\lambda \in \Lambda$ and $T \in M(\lambda)$, set

$$
e_{\lambda}=\sum_{S \in M(\lambda)} C_{S, T}^{\lambda} D_{S, T}^{\lambda} \quad \text { and } \quad L(A)=\left\{\sum_{\lambda \in \Lambda} r_{\lambda} e_{\lambda} \mid r_{\lambda} \in R\right\} .
$$

Now we are in a position to give the main result of this paper.

THEOREM 3.2. Let $A$ be a symmetric cellular algebra with a cellular basis $\left\{C_{S, T}^{\lambda} \mid\right.$ $S, T \in M(\lambda), \lambda \in \Lambda\}$ and the dual basis $\left\{D_{S, T}^{\lambda} \mid S, T \in M(\lambda), \lambda \in \Lambda\right\}$ determined by a symmetrizing trace $\tau$. Then $L(A)$ is an ideal of $Z(A)$ containing the Higman ideal. 


\section{PROOF.}

Step 1. $H(A) \subseteq L(A)$. Clearly, we only need to show that

$$
l:=\sum_{S, T \in M(\lambda), \lambda \in \Lambda} C_{S, T}^{\lambda} C_{U, V}^{\mu} D_{S, T}^{\lambda} \in L(A)
$$

for any $C_{U, V}^{\mu} \in B$, where $\mu \in \Lambda, U, V \in M(\mu)$. We divide $l$ into three parts: $l=$ $l_{\lambda=\mu}+l_{\lambda<\mu}+l_{\lambda \notin \mu}$, where

$$
l_{\lambda=\mu}:=\sum_{S, T \in M(\lambda), \lambda=\mu} C_{S, T}^{\lambda} C_{U, V}^{\mu} D_{S, T}^{\lambda}
$$

and the other two parts are defined similarly.

By Lemma 3.1(7), $l_{\lambda \nless \mu}=0$. We claim that

$$
l_{\lambda=\mu}=\Phi_{\mu}\left(C_{U}, C_{V}\right) e_{\mu} .
$$

In fact, by Lemma 3.1(5), $C_{U, V}^{\mu} D_{X, Y}^{\mu}=0$ if $V \neq Y$. Then from Definition 2.5 and Lemma 3.1(7) we deduce that

$$
\begin{aligned}
l_{\lambda=\mu} & =\sum_{X \in M(\mu)} C_{X, V}^{\mu} C_{U, V}^{\mu} D_{X, V}^{\mu} \\
& =\sum_{X \in M(\mu)} \Phi_{\mu}\left(C_{U}, C_{V}\right) C_{X, V}^{\mu} D_{X, V}^{\mu} \\
& =\Phi_{\mu}\left(C_{U}, C_{V}\right) e_{\mu}
\end{aligned}
$$

This implies that $l_{\lambda=\mu} \in L(A)$.

Now let us consider $l_{\lambda<\mu}$. For arbitrary $\lambda<\mu$, we show that

$$
\sum_{S, T \in M(\lambda)} C_{S, T}^{\lambda} C_{U, V}^{\mu} D_{S, T}^{\lambda}=\sum_{T \in M(\lambda)} r_{(S, T, \lambda),(U, V, \mu),(S, T, \lambda)} e_{\lambda}
$$

Note that

$$
\sum_{S, T \in M(\lambda)} C_{S, T}^{\lambda} C_{U, V}^{\mu} D_{S, T}^{\lambda}=\sum_{S, T \in M(\lambda)}\left(\sum_{\epsilon \in \Lambda, X, Y \in M(\epsilon)} r_{(S, T, \lambda),(U, V, \mu),(X, Y, \epsilon)} C_{X, Y}^{\epsilon}\right) D_{S, T}^{\lambda} .
$$

It follows from Definition 2.5(C2 $\left.3^{\prime}\right)$ that $r_{(S, T, \lambda),(U, V, \mu),(X, Y, \epsilon)}=0$ if $\epsilon \not \lambda$ and Lemma 3.1(7) implies that $C_{X, Y}^{\epsilon} D_{S, T}^{\lambda}=0$ if $\epsilon<\lambda$. Thus

$$
\sum_{S, T \in M(\lambda)} C_{S, T}^{\lambda} C_{U, V}^{\mu} D_{S, T}^{\lambda}=\sum_{S, T \in M(\lambda)} \sum_{X, Y \in M(\lambda)} r_{(S, T, \lambda),(U, V, \mu),(X, Y, \lambda)} C_{X, Y}^{\lambda} D_{S, T}^{\lambda} .
$$

By $\left(\mathrm{C}^{\prime}\right)$ of Definition 2.5, $r_{(S, T, \lambda),(U, V, \mu),(X, Y, \lambda)}=0$ if $X \neq S$ and $C_{X, Y}^{\lambda} D_{S, T}^{\lambda}=0$ if $Y \neq T$ by Lemma 3.1(5). Hence

$$
\sum_{S, T \in M(\lambda)} C_{S, T}^{\lambda} C_{U, V}^{\mu} D_{S, T}^{\lambda}=\sum_{S, T \in M(\lambda)} r_{(S, T, \lambda),(U, V, \mu),(S, T, \lambda)} C_{S, T}^{\lambda} D_{S, T}^{\lambda} .
$$

For arbitrary $S, S^{\prime} \in M(\lambda)$, we have from $\left(\mathrm{C}^{\prime}\right)$ of Definition 2.5 that

$$
r_{(S, T, \lambda),(U, V, \mu),(S, T, \lambda)}=r_{\left(S^{\prime}, T, \lambda\right),(U, V, \mu),\left(S^{\prime}, T, \lambda\right)}
$$


So

$$
\sum_{S, T \in M(\lambda)} C_{S, T}^{\lambda} C_{U, V}^{\mu} D_{S, T}^{\lambda}=\sum_{T \in M(\lambda)} r_{(S, T, \lambda),(U, V, \mu),(S, T, \lambda)} e_{\lambda}
$$

This implies $l_{\lambda<\mu} \in L(A)$. Then we obtain $l \in L(A)$.

Step 2. $L(A) \subseteq Z(A)$. It is sufficient to show that $e_{\lambda} C_{U, V}^{\mu}=C_{U, V}^{\mu} e_{\lambda}$ for arbitrary $\lambda, \mu \in \Lambda, U, V \in M(\mu)$.

On the one hand, by Lemma 3.1(1),

$$
\begin{aligned}
e_{\lambda} C_{U, V}^{\mu} & =\sum_{S \in M(\lambda)} C_{S, T}^{\lambda} D_{S, T}^{\lambda} C_{U, V}^{\mu} \\
& =\sum_{S \in M(\lambda)} \sum_{\epsilon \in \Lambda, X, Y \in M(\epsilon)} r_{(U, V, \mu),(X, Y, \epsilon),(S, T, \lambda)} C_{S, T}^{\lambda} D_{X, Y}^{\epsilon}
\end{aligned}
$$

By a similar method as in Step 1, we get

$$
e_{\lambda} C_{U, V}^{\mu}=\sum_{S, X \in M(\lambda)} r_{(U, V, \mu),(X, T, \lambda),(S, T, \lambda)} C_{S, T}^{\lambda} D_{X, T}^{\lambda} .
$$

On the other hand,

$$
\begin{aligned}
C_{U, V}^{\mu} e_{\lambda} & =\sum_{S \in M(\lambda)} \sum_{\epsilon \in \Lambda, X, Y \in M(\epsilon)} r_{(U, V, \mu),(S, T, \lambda),(X, Y, \epsilon)} C_{X, Y}^{\epsilon} D_{S, T}^{\lambda} \\
& =\sum_{S, X \in M(\lambda)} r_{(U, V, \mu),(S, T, \lambda),(X, T, \lambda)} C_{X, T}^{\lambda} D_{S, T}^{\lambda} .
\end{aligned}
$$

So $e_{\lambda} C_{U, V}^{\mu}=C_{U, V}^{\mu} e_{\lambda}$; that is, $L(A) \subseteq Z(A)$.

Step 3. $L(A)$ is an ideal of $Z(A)$. It suffices to show that, for arbitrary $c \in Z(A)$ and $\lambda \in \Lambda$, the element $c e_{\lambda}$ belongs to $L(A)$; that is, $\sum_{S \in M(\lambda)} C_{S, T}^{\lambda} c D_{S, T}^{\lambda} \in L(A)$. Since $c$ is an $R$-linear combination of elements of $B$, we only need to prove that, for arbitrary $C_{U, V}^{\mu} \in B$, the element $\sum_{S \in M(\lambda)} C_{S, T}^{\lambda} C_{U, V}^{\mu} D_{S, T}^{\lambda}$ belongs to $L(A)$. Clearly, this element is equal to

$$
\sum_{S \in M(\lambda)} \sum_{\epsilon \in \Lambda, X, Y \in M(\epsilon)} r_{(S, T, \lambda),(U, V, \mu),(X, Y, \epsilon)} C_{X, Y}^{\epsilon} D_{S, T}^{\lambda} .
$$

We know that it is equal to $r_{(S, T, \lambda),(U, V, \mu),(X, Y, \epsilon)} e_{\lambda}$ by an argument similar to Step 1 . This implies that $\sum_{S \in M(\lambda)} C_{S, T}^{\lambda} c D_{S, T}^{\lambda} \in L(A)$.

We obtain some properties of $L(A)$ in the following proposition.

PROPOSITION 3.3. With the notation as above:

(1) $L(A)$ is independent of the choice of $\tau$;

(2) if $R$ is a field, then $\operatorname{dim}_{R} L(A) \geq\left|\Lambda_{0}\right|$. 
Proof. (1) Let $\tau, \tau^{\prime}$ be two nonequal symmetrizing traces and $D$ and $d$ the dual bases determined by $\tau$ and $\tau^{\prime}$, respectively. For arbitrary $d_{S, T}^{\lambda} \in d$, it follows from Lemma 2.4 that

$$
d_{S, T}^{\lambda}=\sum_{\varepsilon \in \Lambda, X, Y \in M(\varepsilon)} \tau\left(C_{X, Y}^{\varepsilon} d_{S, T}^{\lambda}\right) D_{X, Y}^{\varepsilon}
$$

Then, by Lemma 3.1,

$$
\begin{aligned}
\sum_{S \in M(\lambda)} C_{S, T}^{\lambda} d_{S, T}^{\lambda} & =\sum_{S \in M(\lambda)} \sum_{\varepsilon \in \Lambda, X, Y \in M(\varepsilon)} \tau\left(C_{X, Y}^{\varepsilon} d_{S, T}^{\lambda}\right) C_{S, T}^{\lambda} D_{X, Y}^{\varepsilon} \\
& =\sum_{S \in M(\lambda)} \sum_{X \in M(\lambda)} \tau\left(C_{X, T}^{\lambda} d_{S, T}^{\lambda}\right) C_{S, T}^{\lambda} D_{X, T}^{\lambda} .
\end{aligned}
$$

By the definition of $\tau$, we have $\tau\left(C_{X, T}^{\lambda} d_{S, T}^{\lambda}\right)=\tau\left(d_{S, T}^{\lambda} C_{X, T}^{\lambda}\right)$. Then, by Lemma 3.1, $\tau\left(d_{S, T}^{\lambda} C_{X, T}^{\lambda}\right)=0$ if $X \neq S$; that is,

$$
\sum_{S \in M(\lambda)} C_{S, T}^{\lambda} d_{S, T}^{\lambda}=\sum_{S \in M(\lambda)} \tau\left(C_{S, T}^{\lambda} d_{S, T}^{\lambda}\right) C_{S, T}^{\lambda} D_{S, T}^{\lambda}
$$

We now need to show that $\tau\left(C_{S, T}^{\lambda} d_{S, T}^{\lambda}\right)$ is independent of $S$. This is clear by the equation $d_{S, T}^{\lambda} C_{S, T}^{\lambda}=d_{S^{\prime}, T}^{\lambda} C_{S^{\prime}, T}^{\lambda}$ for arbitrary $S^{\prime} \in M(\lambda)$.

(2) We only need to find $\left|\Lambda_{0}\right| R$-linear independent elements in $L(A)$. By the definition of $\Lambda_{0}$, for each $\lambda \in \Lambda_{0}$, there exist $S, T \in M(\lambda)$, such that $\Phi_{\lambda}\left(C_{S}, C_{T}\right) \neq 0$. Write $e_{\lambda}=\sum_{U \in M(\lambda)} C_{U, T}^{\lambda} D_{U, T}^{\lambda}$. By Lemma 3.1, we know that the coefficient of $D_{S, T}^{\lambda}$ in the expansion of $C_{S, T}^{\lambda} D_{S, T}^{\lambda}$ is $r_{(S, T, \lambda),(S, T, \lambda),(S, T, \lambda)}=\Phi_{\lambda}\left(C_{S}, C_{T}\right) \neq 0$ and is 0 in the expansion of $C_{U, T}^{\lambda} D_{U, T}^{\lambda}$ for any $U \neq S$. That is, the coefficient of $D_{S, T}^{\lambda}$ in the expansion of $e_{\lambda}$ is not zero. We also know that the coefficient of $D_{S, T}^{\lambda}$ in the expansion of $e_{\mu}$ is zero for any $\mu \not \leq \lambda$. Now let $\sum_{\lambda \in \Lambda_{0}} r_{\lambda} e_{\lambda}=0$ and $\mu$ be a minimal element in $\Lambda_{0}$. Then $r_{\mu}$ must be zero. By induction, we know that $r_{\lambda}=0$ for each $\lambda \in \Lambda_{0}$. This implies that $\left\{e_{\lambda} \mid \lambda \in \Lambda_{0}\right\}$ is $R$-linear independent. That is, $\operatorname{dim} L(A)$ is not less than the number of (representatives of equivalence classes of) simple $A$-modules.

We could define $e_{\lambda}^{\prime}=\sum_{T \in M(\lambda)} D_{S, T}^{\lambda} C_{S, T}^{\lambda}$ for any $\lambda \in \Lambda$ and $L(A)^{\prime}=$ $\left\{\sum_{\lambda \in \Lambda} r_{\lambda} e_{\lambda}^{\prime} \mid r_{\lambda} \in R\right\}$. Then there are analogous results on $L(A)^{\prime}$.

EXAMPLE 3.4. Let $K$ be a field and $Q$ be the quiver

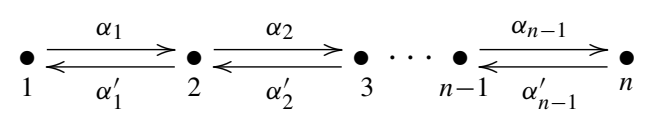

with relation $\rho$ given as follows:

(1) all paths of length $\geq 3$;

(2) $\alpha_{i}^{\prime} \alpha_{i}-\alpha_{i+1} \alpha_{i+1}^{\prime}$, for $i=1, \ldots, n-2$;

(3) $\alpha_{i} \alpha_{i+1}, \alpha_{i+1}^{\prime} \alpha_{i}^{\prime}$, for $i=1, \ldots, n-2$. 
Let $A=K(Q, \rho)$. Define $\tau$ by:

(1) $\tau\left(e_{1}\right)=\cdots=\tau\left(e_{n}\right)=1$;

(2) $\tau\left(\alpha_{i} \alpha_{i}^{\prime}\right)=\tau\left(\alpha_{i}^{\prime} \alpha_{i}\right)=1$, for $i=1, \ldots, n-1$;

(3) $\tau\left(\alpha_{i}\right)=\tau\left(\alpha_{i}^{\prime}\right)=0$.

Then $A$ is a symmetric cellular algebra with a cellular basis

$$
e_{1} ; \begin{array}{cccccccc}
\alpha_{1} \alpha_{1}^{\prime} & \alpha_{1} \\
\alpha_{1}^{\prime} & e_{2} & \alpha_{2} \alpha_{2}^{\prime} & \alpha_{2} & \alpha_{2}^{\prime} & e_{3}
\end{array} ; \quad \ldots ; \quad \begin{array}{ccc}
\alpha_{n-1} \alpha_{n-1}^{\prime} & \alpha_{n-1} \\
\alpha_{n-1}^{\prime} & e_{n} & \alpha_{n-1}^{\prime} \alpha_{n-1}
\end{array}
$$

The dual basis is

$$
\alpha_{1} \alpha_{1}^{\prime} ; \quad \begin{array}{cccccccc}
e_{1} & \alpha_{1}^{\prime} \\
\alpha_{1} & \alpha_{1}^{\prime} \alpha_{1}
\end{array} ; \quad e_{2} \quad \alpha_{2}^{\prime} \alpha_{2} \quad \alpha_{2}^{\prime} \alpha_{2} ; \quad \cdots ; \begin{array}{cccc}
e_{n-1} & \alpha_{n-1}^{\prime} \\
\alpha_{n-1} & \alpha_{n-1}^{\prime} \alpha_{n-1} & e_{n} .
\end{array}
$$

It is easy to see that $L(A)$ is an ideal of $Z(A)$ generated by

$$
\left\{\alpha_{1} \alpha_{1}^{\prime}, \alpha_{1} \alpha_{1}^{\prime}+\alpha_{2} \alpha_{2}^{\prime}, \alpha_{2} \alpha_{2}^{\prime}+\alpha_{3} \alpha_{3}^{\prime}, \ldots, \alpha_{n-2} \alpha_{n-2}^{\prime}+\alpha_{n-1} \alpha_{n-1}^{\prime}, \alpha_{n-1}^{\prime} \alpha_{n-1}\right\}
$$

and $H(A)$ is generated by

$$
\begin{gathered}
\left\{2 \alpha_{1} \alpha_{1}^{\prime}+\alpha_{2} \alpha_{2}^{\prime}, \alpha_{1} \alpha_{1}^{\prime}+2 \alpha_{2} \alpha_{2}^{\prime}+\alpha_{3} \alpha_{3}^{\prime}, \alpha_{2} \alpha_{2}^{\prime}+2 \alpha_{3} \alpha_{3}^{\prime}+\alpha_{4} \alpha_{4}^{\prime}, \ldots,\right. \\
\left.\alpha_{n-3} \alpha_{n-3}^{\prime}+2 \alpha_{n-2} \alpha_{n-2}^{\prime}+\alpha_{n-1} \alpha_{n-1}^{\prime}, \alpha_{n-2} \alpha_{n-2}^{\prime}+2 \alpha_{n-1} \alpha_{n-1}^{\prime}\right\} .
\end{gathered}
$$

Then $\operatorname{dim}_{K} L(A)=n$ since the rank of the following matrix is $n$ :

$$
\left[\begin{array}{ccccccc}
1 & 0 & 0 & 0 & \cdots & 0 & 0 \\
1 & 1 & 0 & 0 & \cdots & 0 & 0 \\
0 & 1 & 1 & 0 & \cdots & 0 & 0 \\
& \cdots & & \cdots & & \cdots & \\
0 & 0 & 0 & 0 & \cdots & 1 & 1 \\
0 & 0 & 0 & 0 & \cdots & 0 & 1
\end{array}\right]_{(n+1) \times n} .
$$

We know that $\operatorname{dim}_{K} H(A)<n$ if char $K$ is a factor of $n+1$ and $\operatorname{dim}_{K} H(A)=n$ otherwise, since the determinant of the following matrix is $n+1$ :

$$
\left[\begin{array}{ccccccc}
2 & 1 & 0 & 0 & \cdots & 0 & 0 \\
1 & 2 & 1 & 0 & \cdots & 0 & 0 \\
0 & 1 & 2 & 1 & \cdots & 0 & 0 \\
& \cdots & & \cdots & & \cdots & \\
0 & 0 & 0 & 0 & \cdots & 2 & 1 \\
0 & 0 & 0 & 0 & \cdots & 1 & 2
\end{array}\right]_{n \times n} .
$$

Then $H(A) \subsetneq L(A)$ if char $K$ is a factor of $n+1$ and $H(A)=L(A)$ otherwise.

There is an interesting orthogonal property of $e_{\lambda}$ which can be stated as follows.

LEMMA 3.5. Keep the notation as above. Then $e_{\lambda} e_{\mu}=0$ for arbitrary $\lambda, \mu \in \Lambda$ with $\lambda \neq \mu$. 
PROOF. For arbitrary $\lambda, \mu \in \Lambda$ with $e_{\lambda} e_{\mu} \neq 0$, there exist $S_{0} \in M(\lambda)$ and $U_{0} \in M(\mu)$ such that

$$
C_{S_{0}, T}^{\lambda} D_{S_{0}, T}^{\lambda} C_{U_{0}, V}^{\mu} D_{U_{0}, V}^{\mu} \neq 0
$$

This implies $D_{S_{0}, T}^{\lambda} C_{U_{0}, V}^{\mu} \neq 0$. Then by Lemma 3.1 there exists some $C_{X, Y}^{\epsilon}$ such that

$$
r_{\left(U_{0}, V, \mu\right),(X, Y, \epsilon),\left(S_{0}, T, \lambda\right)} \neq 0 .
$$

By (C3) of Definition 2.5 this implies that $\lambda \leq \mu$. From $e_{\lambda} e_{\mu}=e_{\mu} e_{\lambda}$, we get $e_{\lambda} e_{\mu} \neq 0$ which implies $\mu \leq \lambda$. Therefore, $\lambda=\mu$ if $e_{\lambda} e_{\mu} \neq 0$.

\section{Semisimple case}

In this section, we consider the semisimple case. We will construct all the central primitive idempotents and give a complete set of primitive orthogonal idempotents for a symmetric cellular algebra.

Firstly, let us recall the definition of Schur elements. For details, see [9].

Let $R$ be a commutative ring with identity and $A$ an $R$-algebra. Let $V$ be an $A$-module which is finitely generated and free over $R$. The algebra homomorphism

$$
\rho_{V}: A \rightarrow \operatorname{End}_{R}(V), \quad \rho_{V}(a) v=a v \quad \text { where } v \in V, a \in A
$$

is called the representation afforded by $V$. The corresponding character is the $R$-linear map defined by

$$
\chi_{V}: A \rightarrow R, \quad a \mapsto \operatorname{tr}\left(\rho_{V}(a)\right),
$$

where $\mathbf{t r}$ is the usual trace of a matrix.

Let $K$ be a field and $A$ a finite-dimensional symmetric $K$-algebra with symmetrizing trace $\tau$. Let $B=\left\{a_{i} \mid i=1, \ldots, n\right\}$ be a basis and $D=\left\{D_{i} \mid i=\right.$ $1, \ldots, n\}$ the dual basis determined by $\tau$. If $V$ is a split simple $A$-module, denote the character by $\chi_{V}$. Therefore,

$$
\sum_{i} \chi_{V}\left(a_{i}\right) \chi_{V}\left(D_{i}\right)=c_{V} \operatorname{dim}_{K} V
$$

where $c_{V} \in K$ is the so-called Schur element associated with $V$. It is nonzero if and only if $V$ is a split simple projective $A$-module [9].

Lemma 4.1 [9, (7.2.7)]. Let A be a split semisimple K-algebra. Then

$$
\left\{e_{V}:=c_{V}^{-1} \sum_{i} \chi_{V}\left(a_{i}\right) D_{i} \mid V \text { is a simple A-module }\right\}
$$

is a complete set of central idempotents which are primitive in $Z(A)$.

Let $R$ be an integral domain and $A$ a symmetric cellular algebra with cellular basis $\left\{C_{S, T}^{\lambda} \mid S, T \in M(\lambda), \lambda \in \Lambda\right\}$. Given a symmetrizing trace $\tau$, the dual basis is $\left\{D_{S, T}^{\lambda} \mid\right.$ $S, T \in M(\lambda), \lambda \in \Lambda\}$. Let $K$ be the field of fractions of $R$. Define $A_{K}:=A \bigotimes_{R} K$. Consider $A$ to be a subalgebra of $A_{K}$ and extend $\tau$ of $A$ to $A_{K}$. Then we can construct all the central idempotents which are primitive in $Z\left(A_{K}\right)$ by $e_{\lambda}$. 
PROPOSITION 4.2. If $A_{K}$ is split semisimple, then $\left\{c_{W(\lambda)}^{-1} e_{\lambda} \mid \lambda \in \Lambda\right\}$ is a complete set of central idempotents which are primitive in $Z\left(A_{K}\right)$.

Proof. The left $A_{K}$-module $W(\lambda)$ is split simple since $A_{K}$ is split semisimple. Then, by Lemma 4.1,

$$
e_{W(\lambda)}=c_{W(\lambda)}^{-1} \sum_{\mu \in \Lambda, U, V \in M(\mu)} \chi_{W(\lambda)}\left(C_{U, V}^{\mu}\right) D_{U, V}^{\mu} .
$$

Note that the character afforded by $W(\lambda)$ is given by the formula

$$
\chi_{W(\lambda)}(a)=\sum_{S \in M(\lambda)} r_{a}(S, S)
$$

for all $a \in A$. Then we get $\chi_{W(\lambda)}\left(C_{U, V}^{\mu}\right)=\sum_{S \in M(\lambda)} r_{(U, V, \mu),(S, T, \lambda),(S, T, \lambda)}$. Then

$$
\begin{aligned}
e_{W(\lambda)} & =c_{W(\lambda)}^{-1} \sum_{\mu \in \Lambda, U, V \in M(\mu)} \sum_{S \in M(\lambda)} r_{(U, V, \mu),(S, T, \lambda),(S, T, \lambda)} D_{U, V}^{\mu} \\
& =c_{W(\lambda)}^{-1} \sum_{S \in M(\lambda)} r_{\mu \in \Lambda, U, V \in M(\mu)} r_{(U, V, \mu),(S, T, \lambda),(S, T, \lambda)} D_{U, V}^{\mu} \\
& =c_{W(\lambda)}^{-1} \sum_{S \in M(\lambda)} C_{S, T}^{\lambda} D_{S, T}^{\lambda} .
\end{aligned}
$$

REMARK 4.3. Clearly, $\left\{e_{\lambda} \mid \lambda \in \Lambda\right\}$ forms a basis of the centre of $A_{K}$ by the above proposition. Jeong et al. in [12, Theorem 3.7] gave a basis of the centres of semisimple Hecke algebras of some finite types, consisting of the $q$-analogues of the conjugacy class sums.

It is helpful to indicate that Dipper and James have given a complete set of primitive orthogonal idempotents of Hecke algebras of general linear groups (see [3, Theorem 5.2]). We could also give a complete set of primitive orthogonal idempotents of symmetric cellular algebras.

COROLlary 4.4. If $A_{K}$ is split semisimple, then

$$
\left\{E_{S}^{\lambda}:=c_{W(\lambda)}^{-1} C_{S, S}^{\lambda} D_{S, S}^{\lambda} \mid \lambda \in \Lambda, S \in M(\lambda)\right\}
$$

is a complete set of primitive orthogonal idempotents of $A_{K}$.

PROOF. For any $\lambda, \mu \in \Lambda, S \in M(\lambda), T \in M(\mu)$, if $\lambda \neq \mu$, the proof of Lemma 3.5 implies that $E_{S}^{\lambda} E_{T}^{\mu}=0$. If $\lambda=\mu$ and $S \neq T$, then $E_{S}^{\lambda} E_{T}^{\lambda}=0$ follows from Lemma 3.1(6). The identities $E_{S}^{\lambda} E_{S}^{\lambda}=E_{S}^{\lambda}$ and $\sum_{\lambda \in \Lambda, S \in M(\lambda)} E_{S}^{\lambda}=1$ are clear by the proof of Proposition 4.2. Finally, the set $\left\{E_{S}^{\lambda} \mid \lambda \in \Lambda, S \in M(\lambda)\right\}$ contains exactly $\sum_{\lambda \in \Lambda} \operatorname{dim}_{K}(W(\lambda))$ elements and therefore primitivity holds.

Note that the centre of $A$ is equal to the intersection of $A$ and the centre of $A_{K}$. We give a necessary condition for an element of the centre of $A_{K}$ being in $A$.

COROLlary 4.5. Let $a_{\lambda} \in K$ for all $\lambda \in \Lambda$ and $a=\sum_{\lambda \in \Lambda} a_{\lambda} e_{\lambda} \in A$. Then $a_{\lambda} c_{W(\lambda)}$ $n_{\lambda} \in R$ for arbitrary $\lambda \in \Lambda$, where $n_{\lambda}$ is the number of elements in the set $M(\lambda)$. 
Proof. For any $\lambda \in \Lambda$, we know that $c_{W(\lambda)}^{-1} e_{\lambda}$ is a central idempotent of $A_{K}$ by Proposition 4.2; that is, $e_{\lambda}^{2}=c_{W(\lambda)} e_{\lambda}$. This implies that $a e_{\lambda}=a_{\lambda} c_{W(\lambda)} e_{\lambda}$. Clearly $a e_{\lambda} \in A$ implies $\tau\left(a e_{\lambda}\right) \in R$. By the definition of the dual basis, $\tau\left(e_{\lambda}\right)=n_{\lambda}$. This completes the proof.

\title{
Acknowledgements
}

The author acknowledges his supervisor Professor C. C. Xi and Dr Wei Hu for many helpful conversations. He also thanks the reviewers for the valuable suggestions which helped to clarify the whole paper.

\section{References}

[1] J. Brundan and C. Stroppel, 'Highest weight categories arising from Khovanov's diagram algebra I: cellularity', arXix: math0806.1532v1.

[2] C. W. Curtis and I. Reiner, Representation Theory of Finite Groups and Associative Algebras (Interscience, New York, 1964).

[3] R. Dipper and G. James, 'Blocks and idempotents of Hecke algebras of general linear groups', Proc. London Math. Soc. (3) 54 (1987), 57-82.

[4] A. Francis, 'The minimal bases for the centre of an Iwahori-Hecke algebra', J. Algebra 221 (1999), $1-28$.

[5] A. Francis, 'Centralizers of Iwahori-Hecke algebras', Trans. Amer. Math. Soc. (7) 353 (2001), 2725-2739.

[6] A. Francis and J. J. Graham, 'Centres of Hecke algebras: the Dipper-James conjecture', J. Algebra 306 (2006), 244-267.

[7] A. Francis and L. Jones, 'On bases of centres of Iwahori-Hecke algebras of the symmetric group', J. Algebra 289 (2005), 42-69.

[8] M. Geck, 'Hecke algebras of finite type are cellular', Invent. Math. 169 (2007), 501-517.

[9] M. Geck and G. Pfeiffer, Characters of Finite Coxeter Groups and Iwahori-Hecke Algebras, London Mathematical Society Monographs, New Series, 21 (Oxford University Press, New York, 2000).

[10] M. Geck and R. Rouquier, 'Centers and simple modules for Iwahori-Hecke algebras', in: Finite Reductive Groups, Luminy, 1994 (Birkhäuser, Boston, MA, 1997), pp. 251-272.

[11] J. J. Graham and G. I. Lehrer, 'Cellular algebras', Invent. Math. 123 (1996), 1-34.

[12] Y.-K. Jeong, I.-S. Lee, H. Oh and K.-H. Park, 'Cellular algebras and centers of Hecke algebras', Bull. Korean Math. Soc. 39 (2002), 71-79.

[13] L. Jones, 'Centers of generic Hecke algebras', Trans. Amer. Math. Soc. 317 (1990), 361-392.

[14] Y. B. Li, 'Radicals of symmetric cellular algebras', Preprint, arXiv: math.RT/0911.3524v1.

[15] G. Malle and A. Mathas, 'Symmetric cyclotomic Hecke algebras', J. Algebra 205 (1998), 275-293.

[16] H. B. Rui and C. C. Xi, 'The representation theory of cyclotomic Temperley-Lieb algebras', Comment. Math. Helv. 79 (2004), 427-450.

[17] C. C. Xi, 'Partition algebras are cellular', Compositio Math. 119 (1999), 99-109.

[18] C. C. Xi, 'On the quasi-heredity of Birman-Wenzl algebras', Adv. Math. 154 (2000), 280-298.

\author{
YANBO LI, Department of Information and Computing Sciences, \\ Northeastern University at Qinhuangdao, Qinhuangdao, 066004, PR China \\ and \\ School of Mathematics Sciences, Beijing Normal University, \\ Beijing, 100875, PR China \\ e-mail: liyanbo707@163.com
}

